\title{
Conductance through the armchair graphene nanoribbons 9-AGNR: Strong dependence on contact to leads
}

\author{
J. A. Vergés, ${ }^{1,2}$ G. Chiappe, ${ }^{2,3}$ Emilio San-Fabián, ${ }^{2,4}$ and E. Louis ${ }^{2,3}$ \\ ${ }^{1}$ Departamento de Teoría y Simulación de Materiales, Instituto de Ciencia de Materiales de Madrid (CSIC), \\ Cantoblanco, 28049 Madrid, Spain \\ ${ }^{2}$ Unidad Asociada del CSIC and Instituto Universitario de Materiales, Universidad de Alicante, San Vicente del Raspeig, \\ 03690 Alicante, Spain \\ ${ }^{3}$ Departamento de Física Aplicada, Universidad de Alicante, San Vicente del Raspeig, 03690 Alicante, Spain \\ ${ }^{4}$ Departamento de Química Física, Universidad de Alicante, 03080 Alicante, Spain
}

(Received 24 October 2017; revised manuscript received 30 July 2018; published 15 October 2018)

\begin{abstract}
The successful use of graphene nanoribbons (GNRs) in a variety of applications in nanoelectronics depends not only on reliable control of their forbidden gaps, but also on the understanding of the effects that contacts to leads may have on their conductance $G$. By combining Landauer's formalism and a simplified version of the embedded cluster method, $G$ through suspended 9-AGNR has been calculated as a function of energy (sample bias) and the strength of the contact between the ribbon and leads attached to both zigzag edges. Green's functions of contacted ribbons have been derived from HF nonspin polarized solutions of the Pariser-Parr-Pople Hamiltonian. It is shown that the $G$ associated with the two quasidegenerate states around the Fermi level, which are strongly localized at the zigzag edges, equals a conductance quantum $G_{0}$ for very weak leads-ribbon coupling, decreasing to zero as that coupling increases. At the Fermi level $G$ is zero for small coupling, increasing up to $G_{0}$ for a value of coupling that depends on the GNR length, and, finally, decreasing to zero for large coupling. Conductance through other energies, starting at $G=0$ for no coupling, increases with coupling to the electrodes up to near one quantum at a pace that may appreciably depend on the particular molecular orbital. These results illustrate the difficulties that may be found in exploring practical uses of GNRs.
\end{abstract}

DOI: 10.1103/PhysRevB.98.155415

\section{INTRODUCTION}

Major improvements in bottom-up technologies are allowing the fabrication of defects-free graphene nanoribbons (GNRs) (actually, mainly armchair ribbons AGNR) with welldefined shape and size [1-13]. This is opening the possibility of controlling the forbidden band gap $[12,14,15]$ and, thus, widen the range of technological applications of graphene $[16,17]$. Measuring the GNR conductance and/or using scanning tunneling spectroscopy (STS) to determine the local density of states (LDOS) have allowed researchers to obtain valuable information on the electronic structure around the HOMO-LUMO gap. Results have been already published for 7-AGNR [1,10-12], 9-AGNR [8], and 13-AGNR [9].

Although most data were taken on ribbons adsorbed on (111) surfaces of fcc metals, especially on $\mathrm{Au}(111)$ [9-12,18], recently several authors have been able to lift off the surface of a single graphene nanoribbon $[1,12,13]$ by controlled pulling of one of the ribbon's ends using a STM. This technique is being applied to a variety of studies of considerable interest [2,4]: (i) keeping one of the GNR's end attached to the STM tip the ribbon was characterized before and after lifting by imaging and spectroscopy [12], (ii) a reliable transfer process of the lifted layer has allowed the investigation of the transistor performance of GNRs [13], and (iii) more recently, transferring the GNRs to a thin $\mathrm{NaCl}$ deposit onto a gold substrate has allowed, according to the authors of Ref. [1], a reliable characterization of the electronic structure of the ribbon. This substantial progress in experimental procedures has allowed a semiquantitative or even quantitative agreement with DFT or model Hamiltonian calculations of the GNR electronic structure [19,20].

Albeit initially most of the theoretical analyses were addressed to investigate the asymptotic behavior of the conductance [17,21], in recent years a strong effort has been addressed to calculate and measure the conductance of small GNR (see above and Refs. [22-26]). It is becoming gradually clearer that a successful use of graphene nanoribbons (GNRs) in a variety of applications in nanoelectronics depend not only on a reliable control of their forbidden gaps, but also on the understanding of the effects that contacts to leads may have on their conductance $G$. Two research groups have recently addressed this issue $[8,26]$. In the first of those two papers the authors showed, by means of DFT calculations, that varying the distance of the STM tip to the GNR may even change the symmetry of the wave function, while in [26] it was concluded that, contacting the leads to the inner atoms of AGNR, the effect of the levels around the Fermi level which are localized at the zigzag edges can be bypassed, triggering in this way a large gap semiconductor behavior.

In this work the conductance $G$ through suspended 9AGNR has been calculated as a function of energy or, equivalently, sample bias, and the strength of the contact between the ribbon and the electrodes attached to both zigzag edges (a parameter that can be varied with a gate voltage). 
Calculations were carried out combining Landauer's formalism and a simplified version of the embedded cluster method [27]. Green's functions of contacted ribbons have been derived from HF nonspin polarized solutions of the PariserParr-Pople Hamiltonian [28-32]. The main results are the following: (i) the $G_{0}$ associated with the two quasidegenerate states around the Fermi level that are strongly localized at the zigzag edges, equals a conductance quantum $G_{0}$ for very weak leads-ribbon coupling, decreasing to zero as that coupling increases; (ii) at the Fermi level $G$ is zero for zero coupling, increasing up to a value around $G_{0}$ for coupling that depends on the GNR length, and, finally, decreasing to zero for large coupling; and (iii) there are also levels that show more standard behavior, i.e., $G$ decreases with coupling.

In addition, we discuss the effects of varying the contacts geometry $[8,26]$ on the GNR conductance and how and why localized orbitals within the bands show a rather standard behavior, as opposed to that of the localized states around the Fermi level.

\section{CONDUCTANCE CALCULATIONS}

At zero temperature and no applied voltage, Landauer's formalism writes the conductance $G$ in terms of the quantum mechanical transmission probability $T$ of electrons at the Fermi level to go from one electrode to the other:

$$
G=\frac{e^{2}}{h} T\left(E_{\mathrm{F}}\right) .
$$

The transmission probability that appears in this equation can be calculated through the expression

$$
T(E)=\operatorname{Tr}\left[\hat{\Gamma}_{\mathrm{L}}(E) \hat{G}(-)(E) \hat{\Gamma}_{\mathrm{R}}(E) \hat{G}^{(+)}(E)\right],
$$

where $\operatorname{Tr}$ denotes the trace over all the orbitals of the cluster and the spin degrees of freedom. The retarded $(+)$ or advanced (-) Green function operators in an orthogonal basis as the one used here (see below) is given by

$$
\hat{G}^{( \pm)}(E)=\left[E \hat{I}-\hat{F}-\hat{\Sigma}^{( \pm)}(E)\right]^{-1} .
$$

The self-energy incorporated in this expression is given by

$$
\hat{\Sigma}^{( \pm)}(E)=\hat{\Sigma}_{\mathrm{R}}^{( \pm)}(E)+\hat{\Sigma}_{\mathrm{L}}^{( \pm)}(E)
$$

where $\hat{\Sigma}_{\mathrm{R}}^{( \pm)}\left(\hat{\Sigma}_{\mathrm{L}}^{( \pm)}\right)$denotes a self-energy matrix that accounts for the semi-infinite left (L) and right (R) electrodes, and the matrices $\hat{\Gamma}_{\mathrm{R}(\mathrm{L})}$ are given by

$$
\hat{\Gamma}_{\mathrm{R}(\mathrm{L})}=i\left(\hat{\Sigma}_{\mathrm{R}(\mathrm{L})}^{(-)}-\hat{\Sigma}_{\mathrm{R}(\mathrm{L})}^{(+)}\right) .
$$

Finally, $\hat{F}$ is the Fock operator associated with the selfconsistent Hamiltonian $\hat{H}$ which in the present case is taken to be the HF paramagnetic solution of Parr-Pariser-Pople (PPP) Hamiltonian [28,29]. As a full description of this issue can be found elsewhere [30-32], here we only remark that the PPP Hamiltonian includes a single $\pi$-like orbital per $\mathrm{C}$ atom (the $s p^{2}$ dangling bonds at each atom in the ribbon were saturated with hydrogen atoms) plus on-site and long range interactions.

In the simplified version of the embedded cluster method used here, independent leads were simulated by purely imaginary self-energies, independent of energy that were attached at the eight atoms of the two zigzag ends (see Fig. 1). This

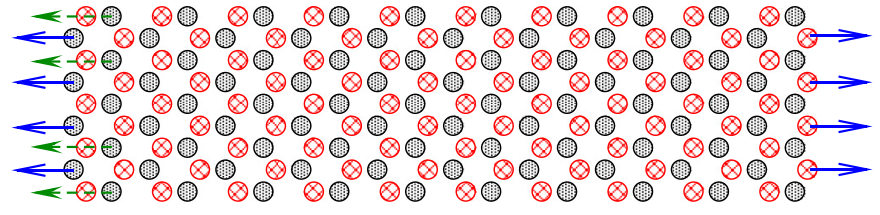

FIG. 1. Contact geometry used in most of the calculations presented here: blue arrows attached at the outer four carbon layers of the left and right zigzag edges of the 9-AGNR10,9. The effect of contact geometry was explored by varying the carbons at which electrodes were attached (for instance, green arrows attached to the second five-carbons layer of the left zigzag edge of a 9-AGNR).

model has been previously used by many authors to investigate, among a large variety of issues, the effects of surface disorder on the conductance of graphene ribbons [21] and of the geometry of metallic contacts on the conductance of carbon nanotubes [33]. The latter authors precisely described the model: The most critical assumption of this model is that leads connected to different carbon atoms are completely independent of each other. This simple model is justified by, as stated in Ref. [33], the fact that the electrodes likely consist of dirty metals without translational symmetry, or, in other words, the probable incoherent nature of contacts [21]. Then we write the self-energy matrices, for 9-AGNR, as

$$
\begin{gathered}
\Sigma_{\mathrm{R}(\mathrm{L})}^{( \pm)}(j, j)=\mp t_{\mathrm{R}(\mathrm{L})} i \\
\text { for } j=1, \ldots, 4(L) \text { and } j=N_{\mathrm{C}}-3, \ldots, N_{\mathrm{C}}(R)
\end{gathered}
$$

and zero otherwise. $N_{\mathrm{C}}$ is the number of $\mathrm{C}$ atoms in the nanoribbon and $t_{\mathrm{R}(\mathrm{L})}$ account for the strength of the right and left contacts of the zigzag atoms to the leads. Hereafter we take $t_{\mathrm{R}(\mathrm{L})}=t$ and, then, $\Gamma_{\mathrm{R}(\mathrm{L})}(i, j)$ matrices are simply derived from Eq. (5).

The GNRs studied here are denominated as AGNR$\{M, N\}, M(N)$ being the number of unit cells in the armchair (zigzag) directions. Successive layers of carbon atoms perpendicular to the armchair direction, contain $\ldots 4,5,5,4, \ldots, 4,5,5,4, \ldots$ carbons, the unit cell being formed by a $[5,4]$ bilayer. Specific calculations were done for $N=9$ and $M=40$ and 60 , which correspond to lengths of 83.2 and $125.5 \AA$, respectively. Then, the total number of carbons in a 9-AGNR will be $N_{\mathrm{C}}=M \times 9$. Besides, we mostly concentrate on energies not far from the Fermi level of a neutral ribbon, albeit the last subsection of Sec. III is devoted to discuss some results for the conductance through a localized molecular orbital within the bands.

\section{RESULTS}

\section{A. Molecular orbitals of the isolated 9-AGNR}

In order to check the validity of our approach for the calculation of the Fock matrix, we have calculated the energies and wave functions of the neutral, isolated, and suspended 9-AGNR. Results for the probability distribution function (PDF) associated with the lowest (highest) three LUMO (HOMO) are depicted in Fig. 2. Note that this ribbon has electron-hole symmetry, a property that is not shared by all AGNR [19]. It is noted that the symmetry of the wave functions coincide with that reported in Ref. [19]. Then, by 


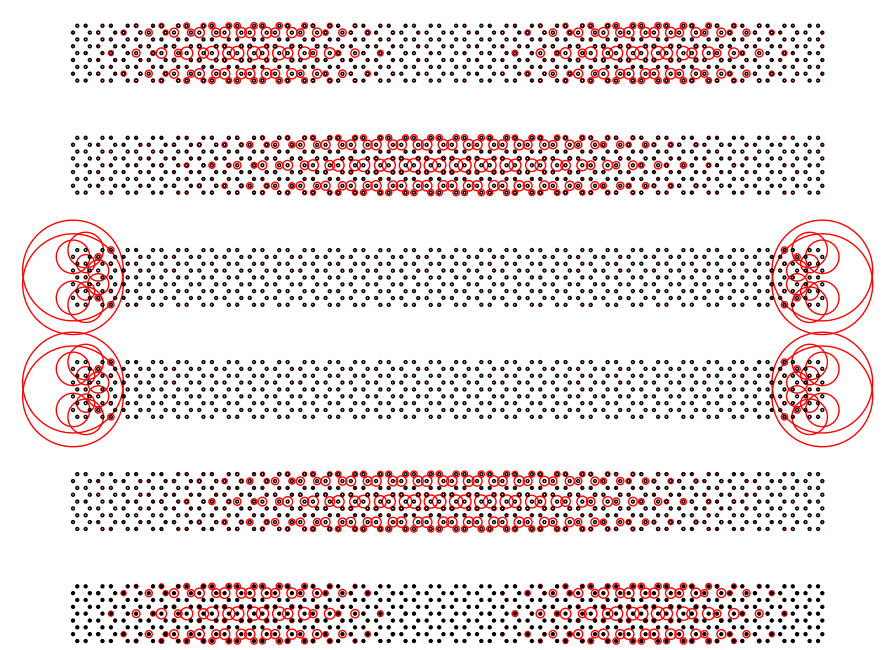

FIG. 2. Probability distribution function (PDF) associated with six electronic states around the Fermi level of a 9-AGNR-\{60,9\}, namely, HOMO-2, HOMO-1, HOMO, LUMO, LUMO+1, and $\mathrm{LUMO}+2$. Their respective energies are $-5.523,-5.452,-3.524$, $-3.406,-1.478$, and $-1.407 \mathrm{eV}$.

recalling the electron-hole symmetry of this ribbon we write $E_{\mathrm{F}}=\left(E_{\mathrm{HOMO}}+E_{\mathrm{LUMO}}\right) / 2=-3.465 \mathrm{eV}$, for the two ribbon lengths considered here. This is to be compared with the results of Ref. [19] $E_{\mathrm{F}}$ for 5-AGNR varying from -3.59 up to $-3.63 \mathrm{eV}$ when the ribbon's length is increased from $M=12$ up to $M=56$, and $-3.66 \mathrm{eV}$ for a 13 -AGNR with $M=3$. The major failure of our approach concerns energy differences of levels around the Fermi level. In particular, while in the present calculation the energy difference between HOMO and LUMO (LUMO+1 and HOMO-1) is $0.12 \mathrm{eV}$ $(3.974 \mathrm{eV})$, the values reported in [19] are a factor 2.5-3.0 smaller, i.e., $0.04 \mathrm{eV}(1.6 \mathrm{eV})$. The latter value is quite close to the recently published experimental result of $1.38 \mathrm{eV}$ [8]. The larger values reported here should be a consequence of the HF approximation used to solve the PPP Hamiltonian, as compared to the state-of-the-art ab initio procedures followed in Ref. [19]. Anyhow, the fact that wave function symmetries are identical, validates the present approach for the study of the problem at hand as symmetry and localization are essential in investigating the effects of the strength of electrodes/ribbon contacts.

\section{B. Conductance versus energy: The odd behavior of $G(\mathrm{LUMO})$ and $G(\mathrm{HOMO})$}

The conductance (given in units of the conductance quantum $G_{0}=2 e^{2} / h$ ) versus energy (from -6 up to $-1 \mathrm{eV}$ ) is depicted in Fig. 3(a) for $t_{\mathrm{R}}=t_{\mathrm{L}}=0.001,0.01,1$, and $2 \mathrm{eV}$, while Fig. 3(b) shows an enlarged picture of the energy range -3.7 up to $-3.5 \mathrm{eV}$ for $t_{\mathrm{R}}=t_{\mathrm{L}}=0.001,0.01,0.1$, and $1 \mathrm{eV}$. Both figures correspond to ribbons 9-AGNR-\{60,9\}. We first draw our attention to energies around the Fermi level $E_{\mathrm{F}}=-3.465 \mathrm{eV}$. The two almost degenerate peaks around $E_{\mathrm{F}}$ give a conductance close to one quantum for very small electrode/ribbon coupling. For large coupling both are smeared out. Actually, already for a coupling as small as $t_{\mathrm{L}}=0.1 \mathrm{eV}$ no signs of those two peaks are noted [see
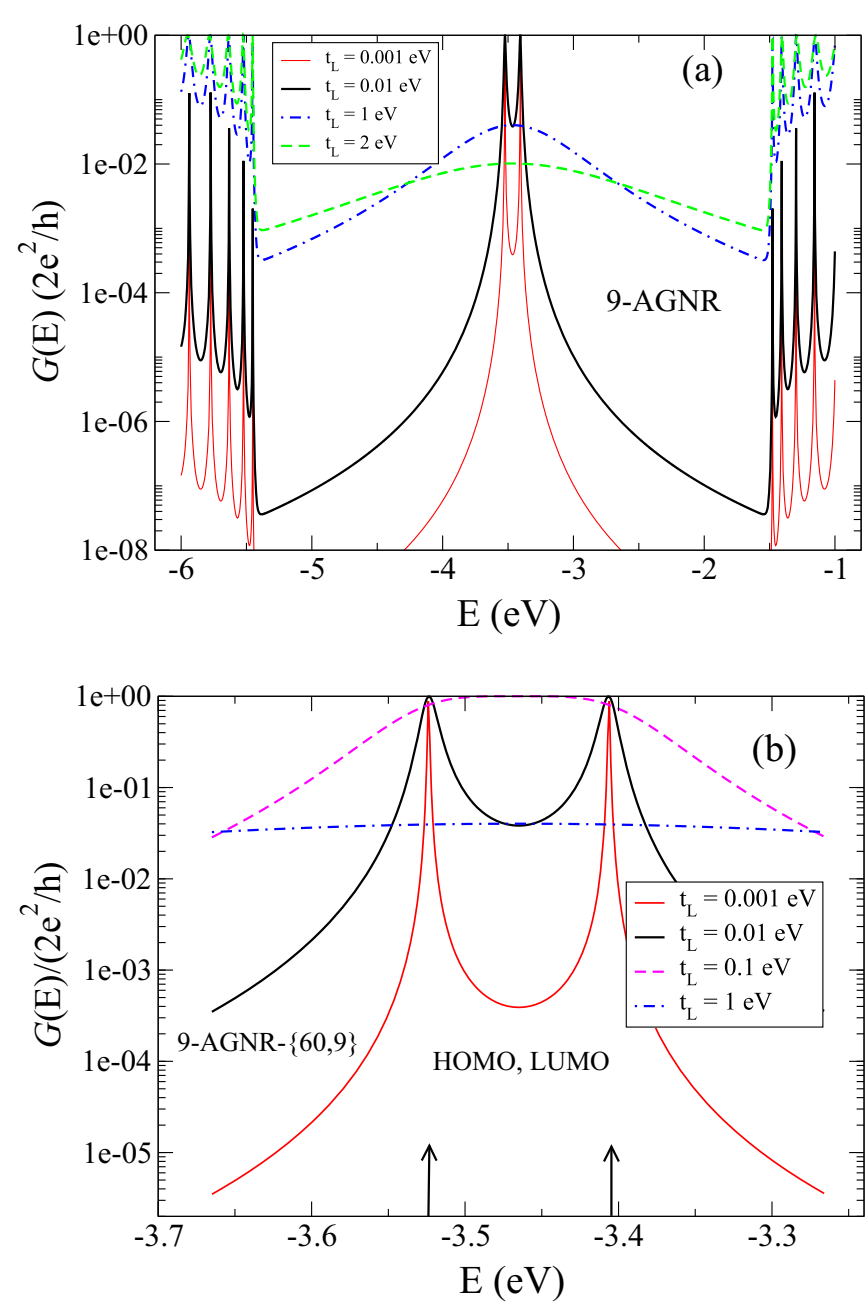

FIG. 3. (a) Conductance (in units of the conductance quantum) through a 9-AGNR- $\{60,9\}$ versus energy for several values of the hopping to leads attached at the two zigzag edges $\left(t_{\mathrm{R}}\right.$ was taken equal to $t_{\mathrm{L}}$ ). For a neutral ribbon the Fermi level $E_{\mathrm{F}}$ lies at $-3.465 \mathrm{eV}$. (b) Shows the region around the Fermi level enlarged.

Fig. 3(b)]. For $t_{\mathrm{L}}=1 \mathrm{eV}$ the conductance is below $0.1 G_{0}$ in the whole range of energies shown in Fig. 3. This is seen even more clearly in Fig. 4 which depicts the conductance as a function of $t_{\mathrm{L}}$. As shown in Fig. 4, the conductance at the HOMO, LUMO, and $E_{\mathrm{F}}$ decreases slightly faster as the ribbon length increases: for large $t_{\mathrm{L}}$, the conductance at HOMO and LUMO follows a power law as $t_{\mathrm{L}}^{-1.67}$ and $t_{\mathrm{L}}^{-1.72}$, for $M=40$ and 60 , respectively. The rather odd behavior of the conductance at the HOMO, LUMO, and $E_{\mathrm{F}}$ is explained by noting that, as $t_{L}$ increases, the perturbation induced by the electrodes penetrates further into the region where the weights of the HOMO and LUMO are negligible. This reduces the conductance through the ribbon. Note that this region widens as the ribbon's length increases. The high localization of both levels is quantitatively illustrated in Fig. 5. Figure 5 depicts the sum of the LDOS, derived from the spectral function, over the nine atoms of successive layers along the ribbon for three values of the ribbon/electrode coupling $t_{\mathrm{L}}$ and two values of the ribbon's length. The results correspond to the energy of the LUMO in each length. It is clearly noted that 


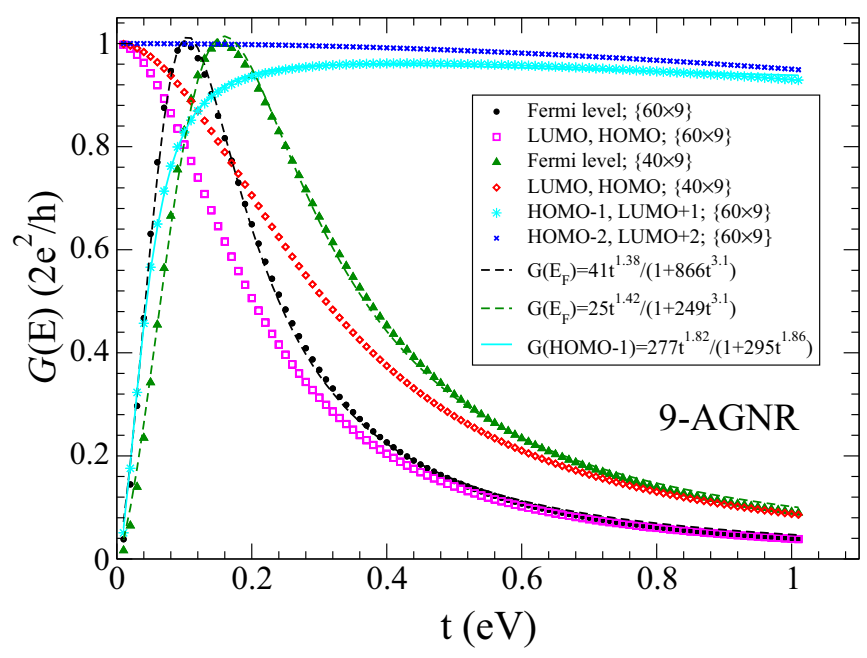

FIG. 4. Conductance through 9-AGNR's of two lengths versus the strength of the ribbon/leads contact (hopping $t_{\mathrm{R}}=t_{\mathrm{L}}$ ). The energy was fixed at the Fermi level, LUMO, HOMO, LUMO+1, LUMO+2, HOMO-1, or HOMO-2 molecular orbitals.

a exponential decay nicely fits the numerical results with a characteristic length of approximately $2 \AA$. This result holds also for the HOMO and does not depend neither on coupling nor on ribbon's length. This supports the explanation offered here for the decay of ribbon conductance, both at LUMO and HOMO energies, with the ribbon/electrode coupling.

At the Fermi level, the conductance does also show a nonstandard behavior. For very small coupling the conductance is smaller than 0.001 quanta increasing up to one quanta for $t_{\mathrm{L}}=0.1 \mathrm{eV}$ [see Fig. 3(b)]. Further increase of ribbon/electrodes coupling diminishes the conductance that becomes of the order of 0.01 quanta for $t_{\mathrm{L}}=2 \mathrm{eV}$. This behavior is most clearly illustrated in Fig. 4 that shows a zero conductance for zero ribbon/coupling, something that strictly holds for all levels varying only the pace at which $G$ raises with $t_{\mathrm{L}}$. Subsequently, the conductance reaches a

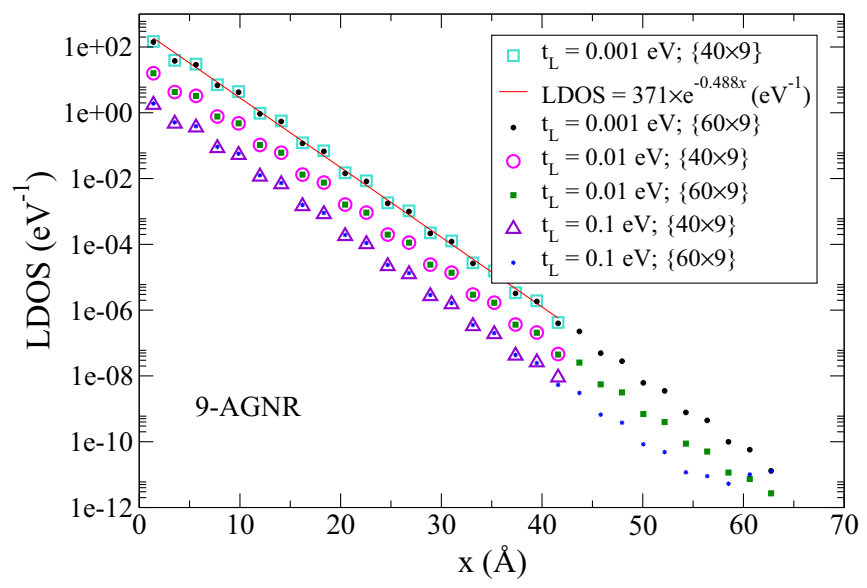

FIG. 5. LDOS sum up over the nine atoms (unit cell along the armchair direction) of successive nine-atom layers along the 9-AGNR- $M \times N$ for $M=40,60$ and $N=9$, versus the position along the length of the ribbon and three values of $t_{\mathrm{L}}$ ( $t_{\mathrm{R}}$ was taken equal to $t_{\mathrm{L}}$ ). The results are identical for HOMO and LUMO.
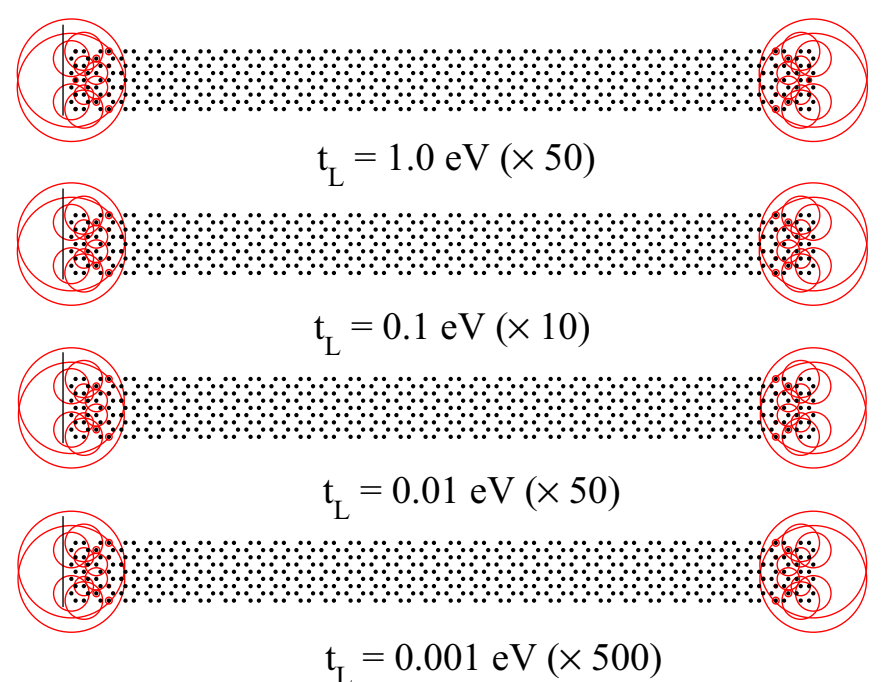

FIG. 6. LDOS at the Fermi level of a 9-AGNR- $\{60,9\}$ derived from the spectral function. The results correspond to four values of the ribbon/electrodes coupling.

maximum for $t_{\mathrm{L}} \approx E_{G}$, where $E_{G}$ is the HOMO-LUMO gap (this is another consequence of the electron-hole symmetry mentioned above). Actually this gap decreases as the length increases between 0.18 and $0.12 \mathrm{eV}$ for $M=40$ and 60 , respectively. Beyond the maximum the conductance follows power laws as $G\left(E_{\mathrm{F}}\right) \approx 0.1 t_{\mathrm{L}}^{-1.68}$ and $G\left(E_{\mathrm{F}}\right) \approx 0.047 t_{\mathrm{L}}^{-1.72}$, for $L=40$ and 60, respectively. This rather odd behavior can again be understood in terms of the strong localization of HOMO and LUMO. For $t_{\mathrm{L}}$ approaching zero the contribution of both HOMO and LUMO to the conductance is a $\delta$ function at their respective energies, being strictly zero at energies inbetween, and, in particular, at $E_{\mathrm{F}}$. Once coupling is switched, the $\delta$ function are smeared out increasing the conductance at $E_{\mathrm{F}}$ up to a maximum close to one quanta. Beyond the maximum the contribution to the conductance of both HOMO and LUMO decreases and so does the conductance at $E_{\mathrm{F}}$. The PDF at the Fermi level versus the ribbon/electrode coupling shown in Fig. 6 adds further support to this interpretation. The symmetry and localization of the LDOS reproduces the PDF of the wave function of the HOMO and LUMO in the isolated ribbon. Varying that coupling only changes the quantitative value of the PDF in a way compatible with the conductance shown in Fig. 4.

\section{Conductance through standard molecular orbitals}

As a final illustration of the relevance that the strength of the ribbon/electrodes contact, in combination with the wavefunction localization and symmetry, have, we discuss the results for the longer ribbon and the HOMO-1 and HOMO-2 levels. The results shown in Fig. 4 are the best for this purpose. It is noted that while the conductance through HOMO-2 is close to one quantum for all $t_{\mathrm{L}}$ shown in Fig. 4, it starts from zero for HOMO-1. Although this can be qualitatively understood by noting that the PDF in HOMO-2 is nonnegligible much closer to the zigzag edges than in HOMO-1, it strongly depends on $t_{\mathrm{L}}$ and on how close is the chosen energy to the actual energy of a particular level. To illustrate 


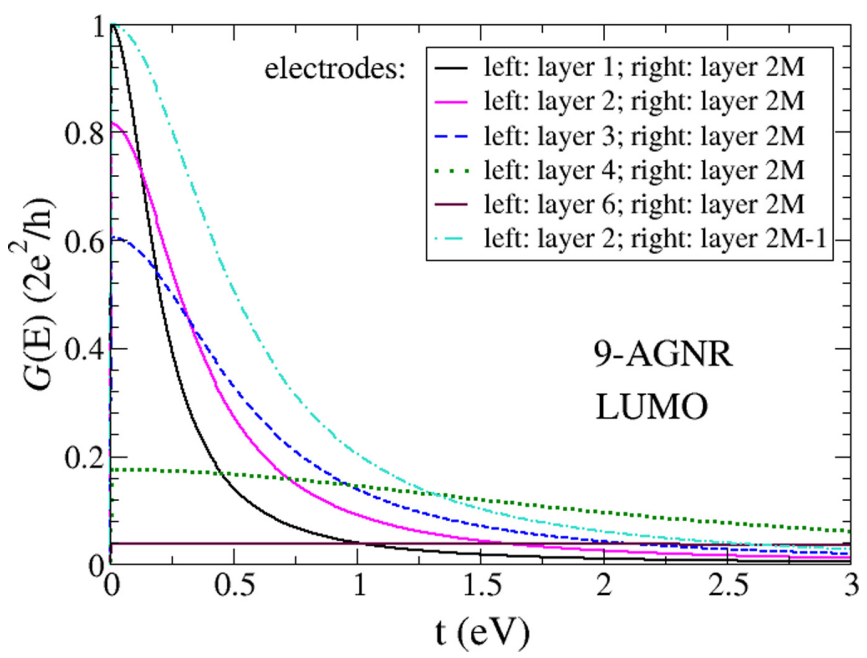

FIG. 7. Conductance through the LUMO of 9-AGNR's of length $M=60$, versus the strength of the ribbon/leads contact (hopping $t_{\mathrm{R}}=t_{\mathrm{L}}=t$ ). The results correspond to different contact geometries.

this issue we consider the cases of LUMO+1 and HOMO-2. While the results reported in Fig. 4 for the LUMO+1 and $t_{\mathrm{L}}=0.01 \mathrm{eV}$, give $0.052 G_{0}$ for $E=-1.4778$, getting closer to the actual eigenvalue gives $0.979 G_{0}$ for $E=-1.47775$ and $G_{0}$ for $E=-1.477752$. On the other hand, if we keep the energy constant and equal to that of the HOMO-2 level $E=$ $-5.52260256 \mathrm{eV}$, we obtain for $t_{\mathrm{L}}=0.000001$ a conductance of $0.0044 G_{0}$ which gradually increases as coupling is raised, i.e., for $t_{L}=0.00001 \mathrm{eV} G=0.31 G_{0}$ and for $t_{\mathrm{L}}=0.0001 \mathrm{eV}$ $G=0.98 G_{0}$. A similar behavior holds for the HOMO and LUMO levels. The weak decrease of $G$ for large $t_{L}$ shown in Fig. 4 can be understood in terms of the perturbation that the electrodes should induce in the wave function. This is the major qualitative difference between those four levels and the HOMO and LUMO as in the latter the sharp decrease of $G$ is not a consequence of a "small perturbation" but rather it is due to their strong localization at the zigzag edges.

\section{Dependence on contact geometry}

The effects of varying the carbons at which electrodes are attached [26] have been also investigated. The most simple case consists of keeping fixed the electrodes at one end, displacing the contacts at the other edge from layer to layer. Let us recall that in our notation the $2 M$ layers along the armchair direction ( $M$ unit cells) are referred to as $l=1,2, \ldots, 2 M-$ $1,2 M$, each pair housing 18 carbon atoms $(=4+5+5+4=18)$. The results for the LUMO are illustrated in Fig. 7. It is noted that, as the left contact is displaced gradually into inner layers of the ribbon (increasing $m$ ), the maximum $G$ (reached at $t=0$ ) decreases while, at the same time, the pace at which $G$ decreases with the strength of the ribbon-leads coupling also diminishes. Both effects are again a consequence of the high localization of LUMO. The conductance obtained with the electrodes attached at the five atoms of layers $m=2$ and $2 M-1$ is also reported in Fig. 7. As it is clearly seen in Fig. 7 this symmetrical configuration gives a conductance higher than those provided by all nonsymmetrical geometries shown

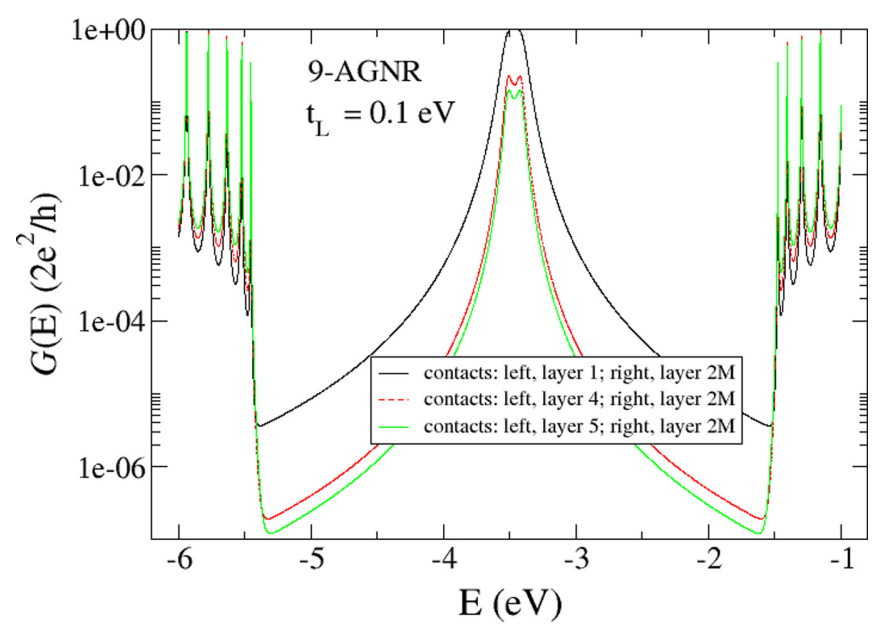

FIG. 8. Conductance (in units of the conductance quantum) through a 9-AGNR-\{60,9\} versus energy for several contact geometries specified in the inset. The results correspond to a ribbon/electrodes coupling of $t_{\mathrm{L}}=0.1 \mathrm{eV}$.

in Fig. 7, being also higher than that obtained with the standard symmetric configuration (electrodes attached at the four atoms of layers $m=1$ and $2 M$ ). A similar calculation of the conductance in the symmetric configuration with electrodes attached at the four atoms of layers $m=2$ and $2 M-1$ based upon a noninteracting Hamiltonian [26] led to a vanishing conductance within the whole main gap presumably due to the much stronger localization of their edge state (mostly confined to the first and last four-atom layers of the ribbon).

Figure 8 shows the conductance through 9-AGNR-\{60,9\} versus energy for several contact geometries as indicated
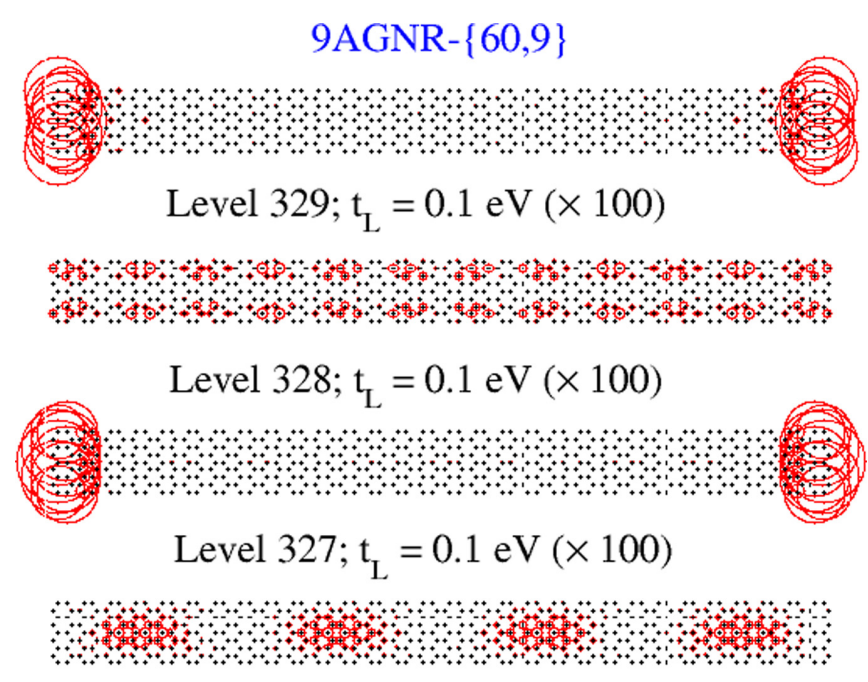

Level 326; $\mathrm{t}_{\mathrm{L}}=0.1 \mathrm{eV}(\times 100)$

FIG. 9. LDOS associated with four molecular orbitals within the conduction band of a 9-AGNR-\{60,9\}. Note that two of them are highly localized at the zigzag edges. The PDF was derived from the spectral function. All results correspond to a ribbon/electrodes coupling of $t_{\mathrm{L}}=0.1 \mathrm{eV}$. 


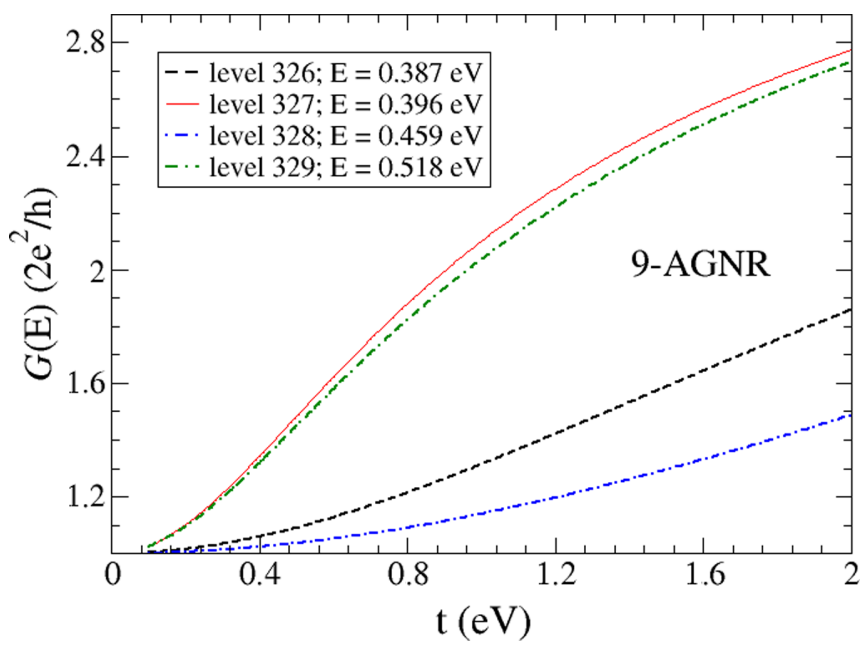

FIG. 10. Conductance through 9-AGNR60,9 versus the strength of the ribbon/leads contact (hopping $t_{\mathrm{R}}=t_{\mathrm{L}}=t$ ). The energies correspond to four molecular orbitals within the conduction band of the ribbon.

in the inset (fixed the electrodes at the right edge of the ribbon and gradually displaced into the inner of the ribbon the electrodes attached to the left edge). The results for a ribbon/electrodes coupling of $t=0.1 \mathrm{eV}$ clearly indicate that the conductance within the whole range of energies is higher for the symmetrical contact geometry. We have checked that this result holds for any value of the coupling.

\section{E. Conductance through localized states within the bands}

We have gone through the bands of the isolated ribbon seeking whether there were further localized states and actually found two in each band (valence and conduction bands) symmetrically placed with respect to the Fermi level. The LDOS associated with the localized molecular orbitals and that to two orbitals close to them, are shown in Fig. 9. The corresponding energies are shown in Fig. 10 (note that the Fermi level, or, center of the main gap, lies at $-3.465 \mathrm{eV}$ ). These localized states lie within size-dependent small gaps (in the case shown in Fig. 9 the gap is $0.07 \mathrm{eV}$ wide) in energy regions of high density of states. To be more specific we note that, whereas within the energy range of $E(\mathrm{LUMO}) \pm$ $0.12 \mathrm{eV}$ only lies a single molecular orbital, six lie in the range $E(327) \pm 0.12 \mathrm{eV}$. This is the origin of the behavior of the conductance shown in Fig. 10: it reaches $G_{0}$ at small $t$ and thereafter it increases, as opposed to the case of the localized states within the main gap.

\section{CONCLUDING REMARKS}

In this work we have investigated the role that the electrode/ribbon contacts play in the conductance of graphene nanoribbons. To this end we have considered neutral, freely suspended 9-AGNR, attached to incoherent metallic electrodes. The results presented here clearly illustrate the major relevance of the strength of the ribbon/electrode contact. The effects we found are not merely quantitative, but rather they are qualitative up to the point that if the contact is not very weak, the conductance through the weakly split HOMO and LUMO may be so small that most experimental techniques would not be able to detect them. The behavior of the conductance at the Fermi level is also rather odd: it shows a maximum for ribbon-electrodes coupling of the order of the HOMO-LUMO gap. These effects are all a consequence of the characteristics of the wave functions that are up to a considerable extent determined by the zigzag ends of the ribbon. Our results, in line with those reported recently [8,26], suggest that special care should be taken when measuring or calculating the electronic structure of armchair graphene nanoribbons. In addition we have studied the effects of the contacts geometry and found how increasing asymmetry decreases conductance. The conductance through states within the bands that are also localized at the zigzag edges have been also studied concluding that the high density of states surrounding the very small gaps where they lie is the main cause of its raise with the ribbon/contact coupling, as opposed to the decrease observed in the states localized in the main gap. The simple approach we have followed is a useful tool to investigate transport properties of GNR at a qualitative, or even semiquantitative, level. In particular, although our calculation of Fock's operator by a HF solution of PPP Hamiltonian cannot of course reach the accuracy of DFT, it is far better than the still used tightbinding method $[8,26]$. The method can be used as a first evaluation of the ribbon performance in relation to a specific technological application.

\section{ACKNOWLEDGMENTS}

Financial support by the Spanish "Ministerio de Ciencia, Innovación y Universidades" (Grants FIS2015-64222-C2-1-P and FIS2015-64222-C2-2-P) and the Universidad de Alicante is gratefully acknowledged.
[1] S. Wang, L. Talirz, C. A. Pignedoli, X. Feng, K. Müllen, R. Fasel, and P. Ruffieux, Nat. Commun. 7, 11507 (2016).

[2] L. Talirz, P. Ruffieux, and R. Fasel, Adv. Mater. 28, 6222 (2016).

[3] W.-X. Wang, M. Zhou, X. Li, S.-Y. Li, X. Wu, W. Duan, and L. He, Phys. Rev. B 93, 241403(R) (2016).

[4] W. Xu and T.-W. Lee, Mater. Horiz. 3, 186 (2016).

[5] C.-N. Yeh, P.-Y. Lee, and J.-D. Chai, Aust. J. Chem. 69, 960 (2016).
[6] C.-S. Wu and J.-D. Chai, J. Chem. Theory Comput. 11, 2003 (2015).

[7] S. Li, C. K. Gan, Y.-W. Son, Y. P. Feng, and S. Y. Quek, Carbon 76, 285 (2014).

[8] L. Talirz, H. Söde, T. Dumslaff, S. Wang, J. R. SánchezValencia, J. Liu, P. Shinde, C. A. Pignedoli, L. Liang, V. Meunier, N. C. Plumb, M. Shi, X. Feng, A. Narita, K. Müllen, R. Fasel, and P. Ruffieux, ACS Nano 11, 1380 (2017). 
[9] Y. C. Chen, D. G. de Oteyza, Z. Pedramrazi, C. Chen, F. R. Fischer, and M. F. Crommie, ACS Nano 7, 6123 (2013).

[10] L. Talirz, H. Sode, J. M. Cai, P. Ruffieux, S. Blankenburg, R. Jafaar, R. Berger, X. Feng, K. Mullen, D. Passerone, R. Fasel, and C. A. Pignedoli, J. Am. Chem. Soc. 135, 2060 (2013).

[11] P. Ruffieux, J. M. Cai, N. C. Plumb, L. Patthey, D. Prezzi, A. Ferretti, E. Molinari, X. L. Feng, K. Mullen, C. A. Pignedoli, and R. Fasel, ACS Nano 6, 6930 (2012).

[12] M. Koch, F. Ample, C. Joachim, and L. Grill, Nat. Nanotech. 7, 713 (2012).

[13] P. B. Bennett, Z. Pedramrazi, A. Madani, Y. C. Chen, D. G. de Oteyza, C. Chen, F. R. Fischer, M. F. Crommie, and J. Bokor, Appl. Phys. Lett. 103, 253114 (2013).

[14] Y.-W. Son, M. L. Cohen, and S. G. Louie, Phys. Rev. Lett. 97, 216803 (2006).

[15] Y.-W. Son, M. L. Cohen, and S. G. Louie, Nature (London) 444, 347 (2006).

[16] A. H. C. Neto, F. Guinea, N. M. R. Peres, K. S. Novoselov, and A. K. Geim, Rev. Mod. Phys. 81, 109 (2009), and references therein.

[17] S. Das Sarma, S. Adam, E. H. Hwan, and E. Rossi, Rev Mod. Phys. 83, 407 (2011), and references therein.

[18] Y. Y. Li, M. X. Cen, M. Weinert, and L. Li, Nat. Commun. 5, 4311 (2014).

[19] A. D. Zdetsis and E. N. Economou, Carbon 116, 422 (2017).
[20] J. A. Vergés, G. Chiappe, and E. Louis, Eur. Phys. J. B 88, 200 (2015).

[21] E. Louis, J. A. Vergés, F. Guinea, and G. Chiappe, Phys. Rev. B 75, 085440 (2007).

[22] F. Molitor, A. Jacobsen, C. Stampfer, J. Güttinger, T. Ihn, and K. Ensslin, Phys. Rev. B 79, 075426 (2009).

[23] C. Motta, D. Sánchez-Portal, and M. I. Trioni, Phys. Chem. Chem. Phys. 14, 10683 (2012).

[24] M. Y. Han, B. Özyilmaz, Y. Zhang, and P. Kim, Phys. Rev. Lett. 98, 206805 (2007).

[25] A. Orlof, J. Ruseckas, and I. V. Zozoulenko, Phys. Rev. B 88, 125409 (2013).

[26] T. Stegmann, J. A. Franco-Villafañe, U. Kuhl, F. Mortessagne, and T. H. Seligman, Phys. Rev. B 95, 035413 (2017).

[27] J. J. Palacios, A. J. Pérez-Jiménez, E. Louis, E. SanFabián, and J. A. Vergés, Phys. Rev. B 66, 035322 (2002).

[28] R. Pariser and R. G. Parr, J. Chem. Phys. 21, 466 (1953).

[29] J. A. Pople, Trans. Faraday Soc. 49, 1375 (1953).

[30] G. Chiappe, E. Louis, E. San-Fabián, and J. A. Vergés, J. Phys.: Condens. Matter 27, 463001 (2015).

[31] J. A. Vergés, E. SanFabián, G. Chiappe, and E. Louis, Phys. Rev. B 81, 085120 (2010).

[32] E. San-Fabián, A. Guijarro, J. A. Vergés, G. Chiappe, and E. Louis, Eur. Phys. J. B 81, 253 (2011).

[33] T. Nakanishi and T. Ando, J. Phys. Soc. Jpn. 69, 2175 (2000). 